Supporting information for

\title{
Deep-Subwavelength Raman Imaging of the Strained GaP Nanowires
}

Vladislav A. Sharov, ${ }^{, \dagger, \dagger}$ Alexey D. Bolshakov, ${ }^{\dagger}$ Vladimir V. Fedorov, ${ }^{\dagger}{ }^{\dagger}$ Stéphanie Bruyere ${ }^{\S}$ George E. Cirlin, ${ }^{\dagger, \|}$ Prokhor A. Alekseev, ${ }^{\ddagger}$ and Ivan S. Mukhin ${ }^{\dagger, \perp}$

$\dagger$ Alferov University (former Saint-Petersburg Academic University), Saint-Petersburg194021, Russia

¥Ioffe Institute, Saint-Petersburg 194021, Russia

†Peter the Great Saint-Petersburg Polytechnic University, Saint-Petersburg 195251, Russia

${ }^{\S}$ Institut Jean Lamour (UMR CNRS 7198), Université de Lorraine, Campus ARTEM, 54000 Nancy, France

"Saint Petersburg Electrotechnical University LETI, Saint-Petersburg 197376, Russia

${ }^{\perp}$ ITMO University, Saint-Petersburg 197101, Russia

E-mail: $\underline{\text { vl_sharov@mail.ru }}$ 
Micro-Raman mapping allows one to study changes in the Raman spectrum in the axial NW direction. The presented maps contain information about the deformation in both axial and radial directions. However, it is worth noting that, in the axial direction, the change in deformation does not occur so quickly toward the radial direction and does not require additional methods providing subwavelength resolution. The axial strain in bent $\mathrm{NW}$ can be characterized via TO mode width $[1,2]$. We analyzed TO width variation along NW length within the obtained map. According to [2], FWHM variation between 11 and $16.5 \mathrm{~cm}^{-1}$ corresponds to strain variation in the range 2-3.5\% (Fig. S1).

(a)

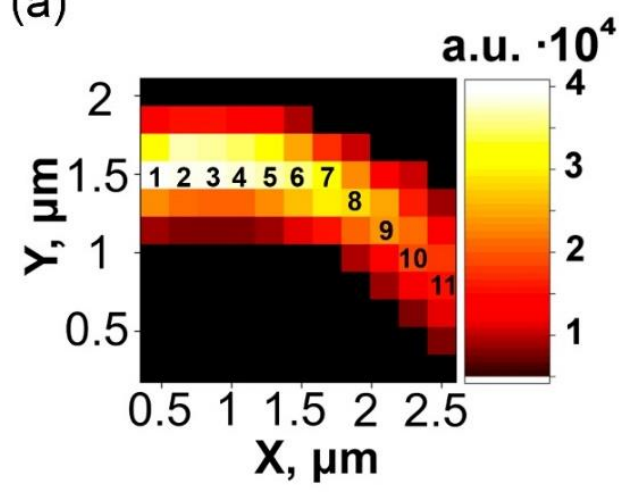

(b)

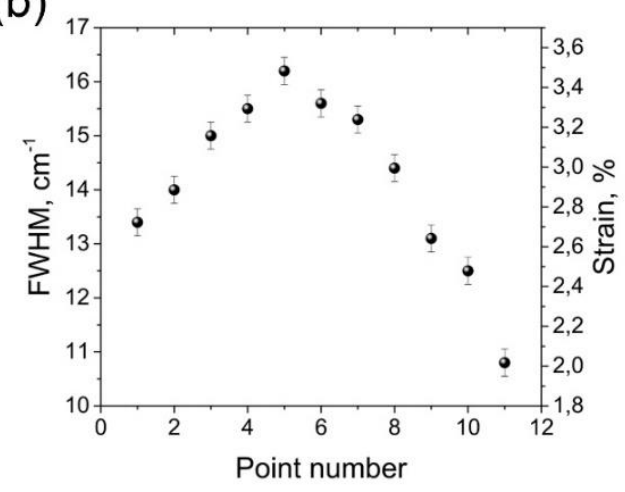

Figure S1. Analysis of strain distribution along the bent nanowire: (a) Integral intensity map of the bent nanowire, (b) TO FWHM values and corresponding bending strains in specified points according to [2].

In order to confirm the reproducibility of the observed strain-induced features in Raman spectrum, two maps of strained NW regions were mapped. Also, 1D profile across another bent NW was obtained. The effect of non-uniform TO broadening and strain-induced shifts was observed across several regions of bent NW with different strain rate (see Fig. S2, S3, S4). All the data was successfully fitted within the introduced model.
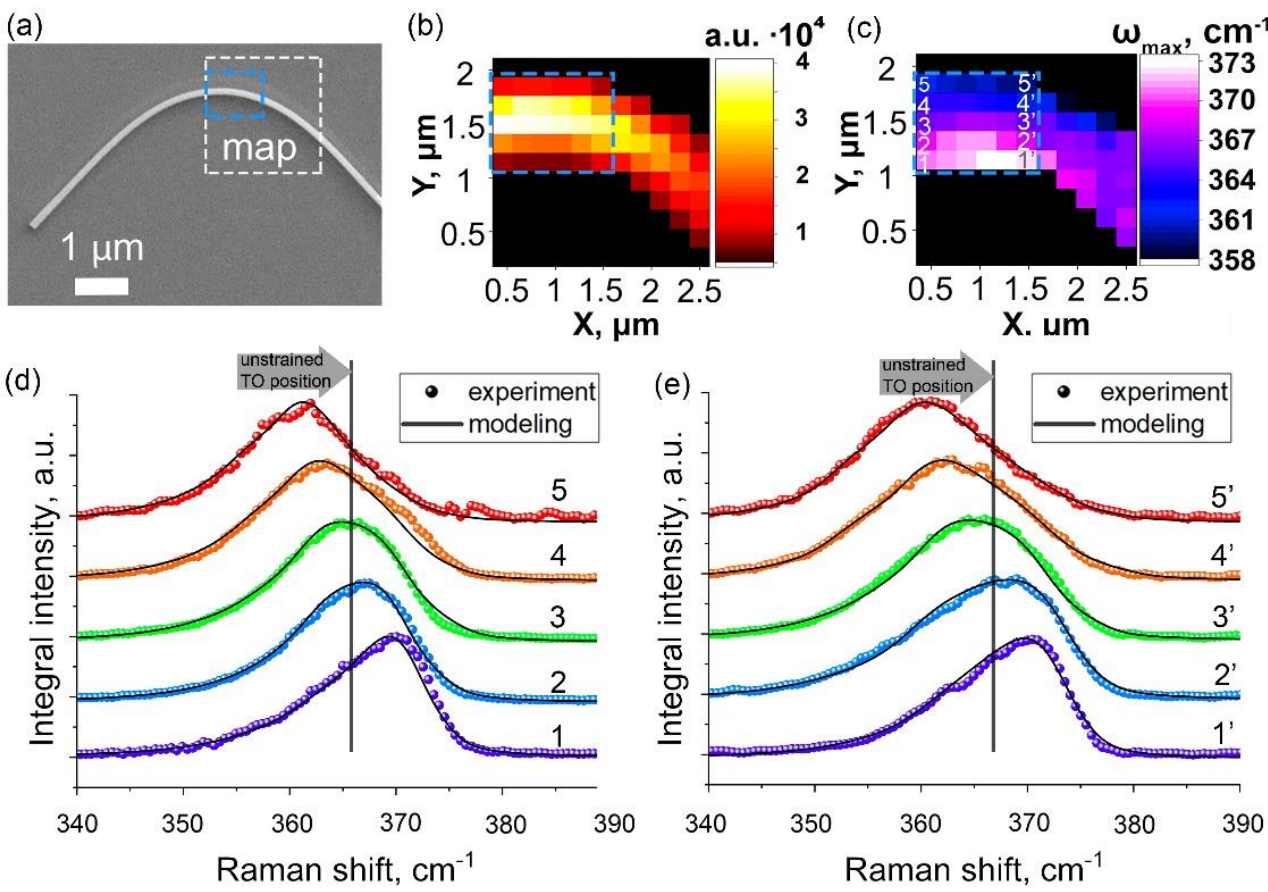

Figure S2. micro-Raman map of bent nanowire: (a) SEM image of the mapped strained region, (b) Integral intensity map, (c) Peak spectral position map, (d, e) Experimental Raman spectra obtained in spots (d) 1-5 and (e) 1'-5' fitted with numerical curves calculated via spatial convolution of theoretical $\mathrm{TO}_{1}, \mathrm{TO}_{2}$ and LA+TA curves with LSF of the optical system. 
(a)

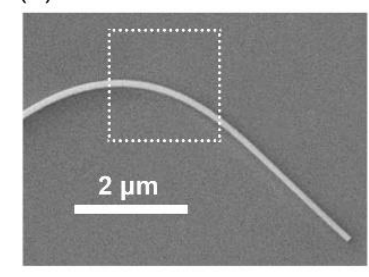

(b)

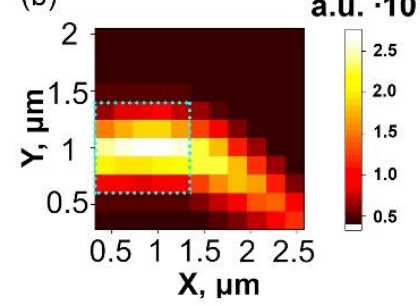

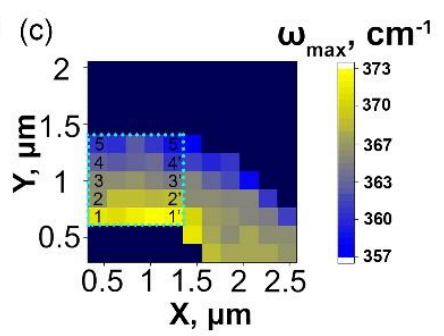
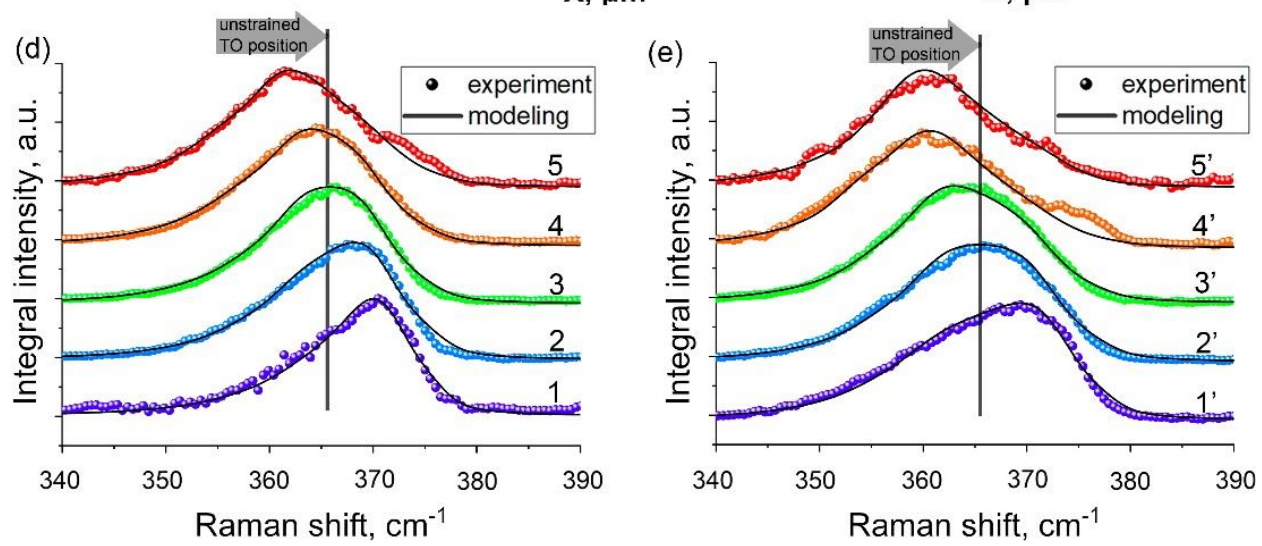

Figure S3. Additional micro-Raman map of bent nanowire: (a) SEM image of the mapped strained region, (b) Integral intensity map, (c) Peak spectral position map, (d, e) Experimental Raman spectra obtained in spots (d) 1-5 and (e) 1'-5' fitted with numerical curves calculated via spatial convolution of theoretical $\mathrm{TO}_{1}, \mathrm{TO}_{2}$ and LA+TA curves with LSF of the optical system.

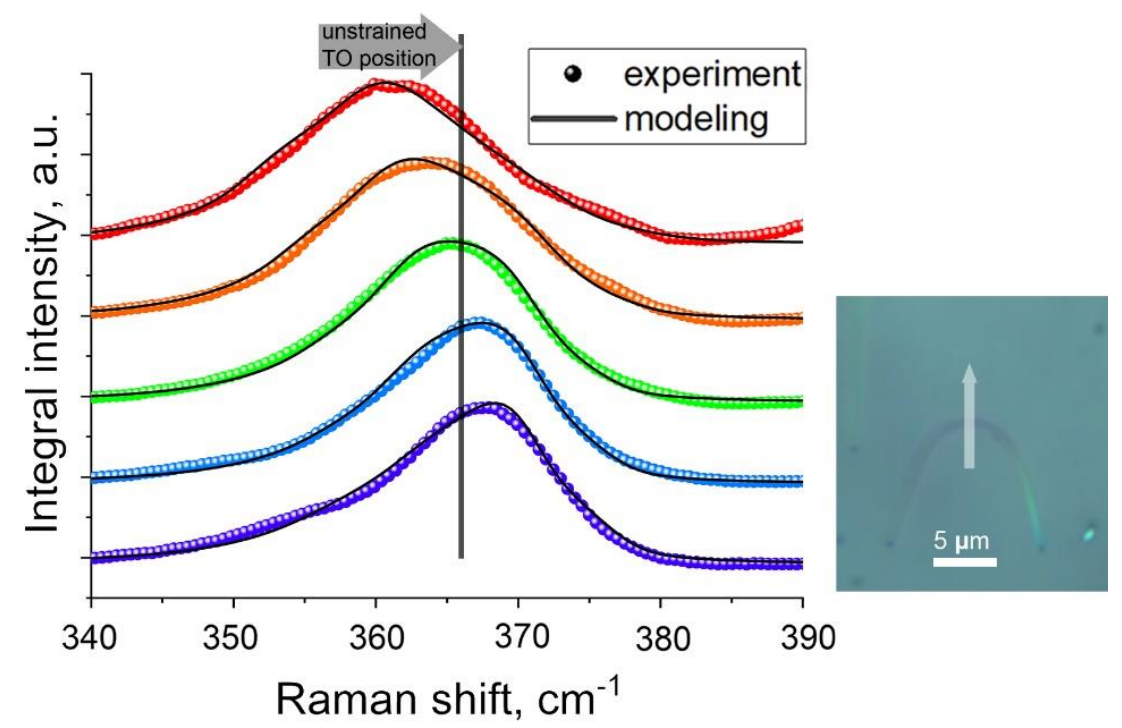

Figure S4. Additional 1D profiling across bent nanowire: smoothed experimental Raman spectra obtained at several pumping spots fitted with numerical curves calculated via spatial convolution of theoretical $\mathrm{TO}_{1}, \mathrm{TO}_{2}$ and LA+TA curves with LSF of the optical system. The inset shows optical image of the bent NW. 


\begin{tabular}{|c|c|c|c|c|c|}
\hline profile & $\xi_{\text {TO1 }}$ & $\xi_{\text {TO2 }}$ & $\xi_{\text {LA+TA }}$ & $\gamma$ & $r / \omega^{2}$ \\
\hline Fig. S2d & 2,5 & 4,4 & 3,2 & 2.08 & -0.39 \\
\hline Fig. S2e & 2,6 & 4,6 & 3,4 & 2.17 & -0.42 \\
\hline Fig. S3d & 2,4 & 4,8 & 3,6 & 2.12 & -0.50 \\
\hline Fig. S3e & 2,1 & 4,2 & 3,1 & 1.86 & -0.44 \\
\hline Fig. S4 & 2,6 & 4,7 & 3,5 & 2.19 & -0.44 \\
\hline Average & 2,44 & 4,54 & 3,36 & 2.08 & -0.44 \\
\hline Error & 0,21 & 0,24 & 0,21 & 0.13 & 0.04 \\
\hline
\end{tabular}

Table S1. Fitting parameters for all the reported profiles.

\section{Fitting details}

Numerical calculations were performed with the use of Python 3.7.

Laser spot was represented with gaussian function $\mathrm{G}(\mathrm{x})$

$$
G(x)=e^{-\frac{\left(x-x_{0}\right)^{2}}{2 c^{2}}}
$$

Where $\mathrm{x}=[0,950] \mathrm{nm}, \mathrm{x}_{0}=450 \mathrm{~nm}, \mathrm{c}=120$.

$\mathrm{TO}_{1,2}$ and LA+TA modes were represented with the following lorentzians:

$$
\begin{aligned}
L_{T O 1}(\omega) & =\frac{1}{\pi \cdot F W H M_{T O 1}\left(1+\frac{\omega-\omega_{(T O 1)}}{F W H M_{T O 1}}\right)^{2}} \\
L_{T O 2}(\omega) & =\frac{1}{\pi \cdot F W H M_{T O 2}\left(1+\frac{\omega-\omega_{(T O 2)}}{F W H M_{T O 2}}\right)^{2}} \\
L_{L A+T A}(\omega) & =\frac{1}{\pi \cdot F W H M_{L A+T A}\left(1+\frac{\omega-\omega_{(L A+T A)}}{F W H M_{L A+T A}}\right)^{2}}
\end{aligned}
$$

Where FWHM $\mathrm{TO}, 2_{2}=4.2 \mathrm{~cm}^{-1}$

$\mathrm{FWHM}_{\mathrm{LA}+\mathrm{TA}}=8.1 \mathrm{~cm}^{-1}$

Strain distribution $\varepsilon(\mathrm{x})$ across NW was set linear, with zero at the NW center and $\pm 3.5 \%$ at the sidewalls.

Peak positions in the presence of strain were calculated as follows:

$$
\omega_{(i)}(\varepsilon)=\omega_{0(i)}-\xi_{i} \cdot \varepsilon(x)
$$

Where $i$ is $\mathrm{TO}_{1,2}$ and $\mathrm{LA}+\mathrm{TA}$

$\omega_{0(\mathrm{TO} 1,2)}=366.1 \mathrm{~cm}^{-1}$ 
$\omega_{0(\mathrm{LA}+\mathrm{TA})}=361 \mathrm{~cm}^{-1}$

All three lorentzians were normalized and summed with the following weights:

$$
L(x, \omega)=0.5 L_{T O 1}(x, \omega)+0.5 L_{T O 2}(x, \omega)+0.25 L_{L A+T A}(x, \omega) \cdot d(\omega)
$$

Here $\mathrm{d}(\omega)$ is two phonon states density taken from (3).

For each $\omega$, the $\mathrm{x}$-component of the resulting array was then convolved with normalized $\mathrm{G}(\mathrm{x})$ :

$\mathrm{C}_{\mathrm{E}}(\mathrm{x})=\mathrm{L}(\mathrm{x}, \omega)^{*} \mathrm{G}(\mathrm{x})$

As a result, $2 \mathrm{D}$ array with the corresponding rows $\mathrm{C}(\mathrm{x}, \omega)$ was evaluated.

Changing $\mathrm{x}$, we choose the pumping spot position ( $\mathrm{x}_{0}$ is the NW center, $\mathrm{x}<\mathrm{x}_{0}$ corresponds to the compressed side, $\mathrm{x}>\mathrm{x}_{0}$ corresponds to the tensed side).

\section{References}

(1) Chen, J.; Conache, G.; Pistol, M.-E.; Gray, S. M.; Borgström, M. T.; Xu, H.; Xu, H.; Samuelson, L.; Håkanson, U. Probing strain in bent semiconductor nanowires with Raman spectroscopy. Nano lett. 2010, $10,1280-1286$.

(2) Im, H. S.; Park, K.; Kim, J.; Kim, D.; Lee, J.; Lee, J. A.; Park, J.; Ahn, J.-P. Strain Mapping and Raman Spectroscopy of Bent GaP and GaAs Nanowires. ACS Omega 2018, 3, 3129-3135.

(3) Ves, S., Loa, I., Syassen, K., Widulle, F., \& Cardona, M. Raman lineshapes of GaP under pressure. physica status solidi (b), 2001, 223(1), 241-245 